\title{
Rede cegonha: Visão de gestantes e profissionais de saúde acerca de sua operacionalização
}

\author{
Flávia Vernaschi Lima ${ }^{1}$, Sílvia F. Rocha Tonhom², Mara Q. Chirelli², \\ Kátia T. Alves Rezende ${ }^{2}$ e Cássia R. F. Biffe Peres ${ }^{2}$ \\ ${ }^{1}$ Departamento Regional de Saúde de Marília - SP, Brasil | fvlmail@uol.com.br
https://orcid.org/0000-0002-1629-4555 \\ ${ }^{2}$ Faculdade de Medicina de Marília - FAMEMA, SP, Brasil | siltonhom@gmail.com; \\ marachirelli@gmail.com; katialvesrezende@gmail.com; c.r.biffe@gmail.com | \\ https://orcid.org/0000-0001-7522-2861; https://orcid.org/0000-0002-7417-4439; \\ http://orcid.org/0000-0002-9022-2680; https://orcid.org/0000-0002-8430-0400
}

\begin{abstract}
Resumo: Introdução: A Rede Cegonha $(\mathrm{RC})$ se propõe a reorganizar a saúde materna e infantil, por meio de ações para o enfrentamento das altas taxas de mortalidade destes segmentos e a formação de uma rede de proteção à mulher e à criança.. Objetivo: analisar a operacionalização da RC em uma Região de Saúde do Estado de São Paulo, a partir da percepção de usuárias e de profissionais de saúde. Método: Estudo qualitativo por meio de entrevistas individuais com 25 usuárias e quatro grupos focais com profissionais da saúde de três municípios de pequeno porte de uma região de saúde. Os dados coletados foram tratados utilizando a análise de conteúdo, modalidade temática. Resultados: Foram abordados os seguintes temas: o ator que exerce o cuidado na perspectiva da gestante; desafios identificados pelas equipes. Conclusão: Embora as ações da RC tenham sido reconhecidas pelas usuárias com alguns apontamentos para melhoria, existem relatos de vulnerabilidade. Ressalta-se, ainda, a utilização das ferramentas propostas pela Política Nacional de Humanização e Política de Humanização do Pré Natal, Parto e Nascimento, cuja atuação participativa e colaborativa dos atores que produzem a saúde possibilita melhores arranjos para o cuidado e, consequentemente, experiências mais seguras e plenas para as mulheres.
\end{abstract}

Palavras-chave: Assistência à Saúde Materno-Infantil; Rede Cegonha; Sistema Único de Saúde.

Stork Network: View of Pregnant Women and Health Professionals about its Operation

\begin{abstract}
The Rede Cegonha (RC) comes as a proposal to reorganize maternal and child health, through actions to face the high mortality rates and the formation of a protection network for women and children. Objective: to analyze the operationalization of RC in a Health Region of the State of São Paulo, from the perception of users and health professionals. Method: Qualitative study through individual interviews with 25 users and four focus groups of health professionals from three small municipalities in a health region. The collected data were treated using content analysis, thematic modality. Results: The following topics were addressed: the perspective of the pregnant Woman of the actor who delivery care; challenges identified by the teams. Conclusion: Although the actions of Rede Cegonha have been observed by users with some notes for improvement, there are reports of vulnerability. It is noticeable that the use of the National Humanization Policy and the Humanization Policy for Pre-Natal, Childbirth and Birth, with the participatory and collaborative work of the actors that produce health, enables better arrangements for care and, consequently, safer and fuller experiences for women.
\end{abstract}

Keywords: Maternal-Child Health Services; Unified Health System.

\section{Introdução}

A Rede Cegonha (RC) no contexto das ações públicas de saúde propõe-se ao enfrentamento das crescentes taxas de mortalidade materna e fetal, do crescente número de partos cesárea, tanto no âmbito do Sistema Único de Saúde (SUS) como na saúde suplementar e privada, e da fragilização do cuidado pré-natal que direciona as ações tanto das mulheres como das equipes de saúde a escolhas baseadas em conhecimentos pobres em evidências científicas, cercadas de mitos e medos (Brasil, 2011a). 
A organização da RC se faz a partir de quatro (4) componentes: (i) Pré-Natal; (ii) Parto e Nascimento; (iii) Puerpério e Atenção Integral à Saúde da Criança; e (iv) Sistema Logístico: Transporte Sanitário e Regulação.

Cada componente se desenvolve em um cenário específico, sendo o Pré-Natal desenvolvido principalmente no território da Atenção Básica (AB); o Parto e Nascimento desenvolve-se principalmente na atenção hospitalar, tanto nas maternidades de baixo como de alto risco, nos Centros de Parto Normal e Casas de Parto; o Puerpério e Atenção Integral à Saúde da Criança ocorre, na sua grande maioria, de volta ao território da $A B$, podendo compartilhar 0 atendimento especializado nas necessidades mais complexas e o componente Sistema Logístico perpassa todos os cenários, inclusive das Urgências.

Embora o acesso ao pré-natal seja praticamente universal no Brasil com alcance dos indicadores de número de consultas de pré-natal executadas, a qualidade dessa atenção não é suficiente para promover um bom cuidado (Brasil, 2011b).

O cenário foco-dessa pesquisa é a RC de uma região de saúde (RS) composta por 19 municípios, pertencente a uma das Redes Regionalizadas de Atenção em Saúde (RRAS), denominada RRAS 10 (correspondente ao Departamento Regional de Saúde IX - DRS IX), que dividem organizativamente o sistema de saúde do Estado de São Paulo, Brasil (São Paulo, 2011).

Considerando o Plano de Ação Regional da RC da RRAS, aprovado nas instâncias estaduais e nacionais (São Paulo, 2012), identifica-se o recrudescimento da mortalidade materna, destacando-se que na RS desse estudo de 22,15 por 100.000 nascidos vivos em 2010, esse número avança para 52,72 em 2016 (Brasil, 2018).

A construção e desenvolvimento da $\mathrm{RC}$, aliados ao desenvolvimento das Redes de Atenção, ancorados nos princípios da Política Nacional de Humanização (Brasil, 2008) e, por consequência, nos princípios do SUS, implicam a transformação dos modelos hierarquizados para modelos horizontais dos pontos de atenção, e promoção da autonomia dos sujeitos atores da produção de saúde.

No entanto, conforme os indicadores apontados anteriormente, acredita-se que poucos avanços ocorreram, evidenciando, ainda, um modelo fragmentado e não efetivo à essa população.

Diante disso, tem-se como inquietação compreender a organização do cuidado do prénatal na RC, de forma a identificar as fragilidades na implementação dessa política de saúde e a compreensão das ferramentas propostas.

Assim, objetiva-se analisar a operacionalização da RC em uma Região de Saúde do Estado de São Paulo, a partir da percepção de usuárias e de profissionais de saúde.

\section{Metodologia}

Trata-se de uma pesquisa qualitativa que trabalha com o universo dos significados, dos motivos, buscando a compreensão das crenças, dos valores e das atitudes, das percepções e interpretações que as pessoas fazem a respeito do modo de viver e pensar, propiciando a construção de novas abordagens e criação de novos conceitos a partir da investigação. (Minayo, 2014 e 2015)

A investigação foi realizada em três municípios que pertencem ao DRS IX, definidos por amostra de conveniência considerando o número de atendimentos e encaminhamentos ao hospital de referência e designados como $A M, A B E C M$. Assim, $A M$ e $C M$ são os municípios que mais encaminham à referência, sendo que $A M$ apresenta cobertura de $100 \%$ de ESF; o município BM que conta com 100\% de cobertura de $\mathrm{AB}$ e $100 \%$ de ESF, o que menos encaminha; e CM tem AB com Estratégia Saúde da Família (ESF) e Unidade Saúde da Família (UBS) tradicional, apresentando cobertura de aproximadamente $70 \%$ de $A B$. 
Para participação na pesquisa, todas as gestantes inscritas e com pré-natal em curso, na rede pública ou na rede privada, foram convidadas pelos profissionais das equipes, tendo sido entrevistadas as que aceitaram, e quanto aos profissionais, foram incluídos por participação na equipe de saúde e por aceite na participação no estudo.

A coleta de dados foi realizada por meio das técnicas de entrevista e de grupo focal. A entrevista na pesquisa social é descrita em Minayo (2015) como uma técnica da pesquisa de campo para coleta de informações que tem como objetivo construir informações sobre o objeto de pesquisa e não prescinde da interação entre 0 pesquisador e os sujeitos pesquisados. Dentre as modalidades propostas pela autora, a entrevista semiestruturada combina perguntas fechadas e abertas e convida 0 interlocutor a discorrer sobre o assunto sem se prender à questão formulada.

O roteiro de perguntas semiestruturadas foi desenvolvido com o olhar para os dispositivos e ferramentas da RC. Foram entrevistadas individualmente 25 usuárias, no período de agosto a novembro de 2019 , com duração média de $13 \mathrm{~min}$, em locais que variaram, à escolha da entrevistada, entre a própria residência e a unidade de saúde.

Para a realização dos grupos focais com as equipes de saúde, foram convidados os componentes da equipe mínima, das unidades dos municípios onde as gestantes residiam: Agente Comunitário de Saúde (ACS), Enfermeiro, Médico, Auxiliar/Técnico de Enfermagem e Dentista, tendo em duas unidades participado também Agente de Controle de Endemias (ACE) e Auxiliar de Limpeza, pela compreensão que a equipe tinha de pertencimento.

Minayo (2014) destaca a praticidade de reunir muitos atores em um mesmo momento, a possibilidade de desvelar consensos e dissensos, importante para aprofundar o debate do grupo. A análise do grupo focal pode fornecer subsídio para a compreensão dos conteúdos emergidos das entrevistas e estas, por sua vez, aos dados do grupo, para identificação dos conceitos percebidos e construídos pelos atores profissionais da saúde e usuárias sobre rede de atenção e rede cegonha, interesse do estudo que ora conduzimos.

Foram formados quatro grupos, identificados de acordo com a nomenclatura utilizada para os municípios: um grupo nos municípios AM e BM cada, EqAM e EqBM respectivamente e dois grupos no município CM, por este ter uma equipe de UBS Tradicional e uma equipe de ESF, sendo denominados EqCM1 e EqCM2. Os grupos somente foram realizados após a assinatura do Termo de Consentimento Livre e Esclarecido (TCLE).

Estes encontros ocorreram no período de agosto a novembro de 2019 na própria unidade de saúde e em horário de trabalho, sem prejuízo ao trabalhador, com uma média de $33 \mathrm{~min}$ de duração. O roteiro, continha questões norteadoras abordando aspectos referentes a organização e a operacionalização da rede cegonha, as fragilidades e as potências por eles identificados.

As entrevistas e os grupos focais foram registrados em áudio por meio de gravador digital ou aplicativo em telefone móvel e transcritas posteriormente.

Para garantia de sigilo, as entrevistas foram numeradas e codificadas, sendo referenciadas de acordo com o município, $\mathrm{AM}, \mathrm{BM}$ e $\mathrm{CM}$, em ordem numérica: AM1, AM2 [...]; BM1, BM2 [...] e CM1, CM2 [...].

Para realização da pesquisa, obteve-se autorização dos respectivos gestores municipais e estaduais das instituições envolvidas e a submissão ao Comitê de Ética em Pesquisa da Faculdade de XXX, com parecer favorável, CAAE 02880118.4.0000.5413, por № 3.739.674.

A análise dos dados foi realizada pela Análise de Conteúdo, modalidade temática, segundo o referencial de Minayo (2015). 
Sendo assim, as transcrições das entrevistas e dos grupos focais passaram por exaustiva leitura para exploração, identificação e organização dos conteúdos das falas, reconhecimento dos núcleos de sentido, e posterior categorização e definição dos temas, de acordo com o procedimento metodológico da análise de conteúdo. O processo de codificação foi realizado pela pesquisadora e duas docentes que fizeram a validação. Obteve-se denso material que nesse artigo será abordado aspectos sob o olhar da gestante e profissionais acerca do tema: o ator que exerce o cuidado e os desafios identificados pelas equipes.

\section{Resultados}

\subsection{O Ator que Exerce o Cuidado na Perspectiva da Gestante}

Os relatos trouxeram percepções sobre o cuidado no pré-natal e do profissional que o realiza, com consultas periódicas intercaladas entre o enfermeiro e o médico, e de procedimentos realizados especificamente pelo profissional enfermeiro e outros a percepção do cuidado feito especificamente pelo médico:

"Uma [consulta] com o enfermeiro, uma com o médico" (BM2).

"A primeira com enfermeira, a segunda com médico, a terceira com enfermeira, a quarta com médico ginecologista, a quinta com enfermeira" (BM14).

"Já passei [com a enfermeira]" (AM1).

"[Na pré consulta] mede a pressão, pergunta se tem alguma queixa, peso" (AM3).

"Sempre que venho faz a pré consulta primeiro, só peso e pressão, depois passa com a médica" (AM2).

"Quando eu descobri [a gravidez] foi com a enfermeira [...] ela preencheu a carteirinha" (BM12).

Concernente aos profissionais atenderem as necessidades de informações, identificouse satisfação tanto no serviço privado quanto no público e orientações que geraram dúvidas.

"No particular eles conversam muito, é 100\% diferente do SUS, eles ficam muito com você" (BM12).

"A médica aqui conversa bastante. Tá de parabéns, fui atendida muito bem no posto. Ganhamos até banheira, fralda..." (CM3).

"Aqui é bem-feitinho, melhor que o particular" (BM3).

Outros relatos expuseram, todavia, algumas fragilidades nas informações de que dispunham e o quão vulneráveis as gestantes estavam por estas serem poucas ou desqualificadas:

"Não vou conseguir amamentar [por causa da incompatibilidade Rh (SIC)], não vou conseguir fazer várias coisas" (AM1).

"Não recebi orientação [sobre os tipos de parto]" (BM2).

"Eles não falaram muita coisa [sobre o parto normal] o que eu sei é o que eu ando lendo" (CM2). 


\subsection{Desafios Identificados pelas Equipes}

Em relação aos desafios para a operacionalização da $R C$, as equipes trazem aspectos relacionados ao acompanhamento do processo em Rede, além de dificuldades na organização do processo de trabalho e outros relacionados a adesão das gestantes.

No que se refere ao processo de gestão tem-se dificuldades em acompanhar o processo de cuidado, embora, acreditam que desenvolvam muitas ações.

"A gente faz muito, vai ver a efetividade, não consegue acompanhar, então, esse monitoramento, a gente não tem" (EQAM).

Quanto ao processo de trabalho, apontam-se fragilidades como a sobrecarga de trabalho, o número reduzido de profissionais para atender às necessidades da população e a rotatividade do profissional médico:

"Meu desafio é desenvolver, no pouco tempo que a gente tem, [...] as várias atividades" (EQAM).

"A quantidade de população é grande e a quantidade de profissional não dá conta" (EQCM2).

"Não tem um profissional [médico] fixo. Quando o paciente "acostuma" com o profissional, o profissional acostuma com o paciente... Daí tem que começar tudo de novo" (EQCM2).

As gestantes também são identificadas, por profissionais da equipe de saúde, como responsáveis por fragilizar o cuidado as mesmas.

"Gestante, a gente convida, mas não vem. Resistência" (EQBM).

"A população exige muito da gente" (EQBM).

"As gestantes não têm compromisso" (EQCM1).

Outro desafio apontado pelos profissionais diz respeito a descontinuidade do cuidado no sentido de ausência de retaguarda na sequência da Rede e incongruência de orientações com o serviço hospitalar.

"Aqui na unidade tem assistência boa, trata, instrui, só que lá na frente não tem um ultrassom, um parto digno. Não tem como" (EQAM).

"A mulherada vai lá, tem o bebê, volta aqui contando horrores do que passou lá no parto" (EQCM1).

"Mas na amamentação, tá com problema, tem os industrializados, já sai do hospital com a receita do pediatra" (EQAM).

\section{Discussão}

Em relação ao cuidado do pré-natal, tem-se o atendimento realizado por médico e por enfermeiros. Destaca-se que a ação da enfermagem se amplia no pré-natal de baixo risco na $A B$, principalmente no âmbito da $E S F$, pois cabe ao profissional enfermeiro o cuidado compartilhado e alternado das consultas com o médico, tendo um papel ativo na escuta, avaliação, prescrição e orientação, conforme os documentos técnicos estabelecidos pelo Ministério da Saúde (2012) e Conselho Federal de Enfermagem (COFEN, 2016 e 2017). O papel do enfermeiro é essencial para a ampliação das ações de humanização na assistência, construindo vínculos, estabelecendo relações de confiança e organizando o processo de trabalho (Gomes et al. 2019). 
Pesquisa realizada com gestantes aponta que enfermeiros realizam consultas mais acolhedoras e que privilegiam a escuta (Livramento, 2019a). Dessa forma, a consulta de enfermagem de forma alternada com a consulta médica deve ser uma ação mais presente nos serviços de saúde, ou seja, não ser uma atividade somente de pré consulta médica, como também citada na presente investigação.

No cenário pesquisado, identificam-se incompreensões quanto às informações recebidas pelas gestantes. Nesse sentido, Cardelli et al. (2016) apontam as expectativas e satisfação manifestas pelas gestantes relacionadas ao cuidado do pré-natal e concluem que há necessidade de informação, escuta e acolhimento, para além da realização de consulta médica e exames complementares, principalmente nos estratos das primíparas e das gestantes qualificadas como de alto risco.

Pires et al. (2016) consideram as dificuldades observadas na $A B$, mesmo com a implantação da ESF, como os cenários complexos do trabalho vivenciados no território e as condições disponíveis para sua realização, interferindo na qualidade e segurança do resultado do cuidado, sendo aspectos importantes para a análise dos relatos das equipes sobre os desafios identificados.

Com relação à rotatividade dos médicos, Pierantoni et al. (2015) reportam índices deste fenômeno, observados tanto nas UBS tradicionais como nas Unidades com ESF. Municípios com indicadores econômicos baixos, a concorrência do mercado, os fatores demográficos, geográficos, a estrutura e o acesso aos níveis de atenção de maior complexidade e, por fim, a satisfação para o trabalho estão entre os pontos discutidos. Em estudo comparativo, Elias et al. (2006) encontraram diferenças importantes, tendendo à melhor na avaliação para as unidades cujo modelo de atenção estivesse pautado pela ESF.

À luz destas reflexões, compreende-se que tanto nas unidades cujo trabalho é pautado no modelo de atenção da ESF como nas UBS tradicionais, estas convivem com subdimensionamento de profissionais, sobrecarga de trabalho, entre outros problemas. Esse contexto com descontinuidade no acompanhamento das gestantes, principalmente por parte da alta rotatividade dos médicos, não contribui para um cuidado integral, em Rede, na medida em que o foco muitas vezes está na queixa momentânea e não nas necessidades que possam se constituir considerando a idade gestacional, número de filhos, contexto socioeconômico, promoção da autonomia das gestantes nas escolhas ao longo do pré-natal, dentre outras.

Os programas da ESF e da PNH (Brasil, 2006 e 2008) trouxeram, no bojo das formulações, novos paradigmas para construção da autonomia de trabalhadores e usuários. Estas ferramentas trazem novas formas de relação entre estes atores, permitindo novos arranjos, transcendendo o lugar-comum da subjugação dos sujeitos para ações vivas e transformadoras (Freire, 2014). Terra e Campos (2019) ressaltam que o papel de expectadores nos apequena diante do trabalho, podendo trazer tristeza e alienação.

Capturadas pelos grupos focais, as frases responsabilizando o usuário pelo não cuidado, são recorrentes nas equipes de saúde. De fato, e em última instância, as pessoas são responsáveis pelas próprias escolhas, no entanto, necessita-se de investimentos para possibilitar autonomia para tomada de decisão, como atores-no seu processo de cuidar.

Os profissionais direcionam às gestantes a responsabilidade pela descontinuidade no cuidado, mas não conseguem perceber que não há uma proposta de gestão a partir de indicadores e reflexão sobre o próprio processo de trabalho, o qual pode não estar atendendo as necessidades de saúde delas. Mesmo que as gestantes estejam satisfeitas com o atendimento, conforme destacado em algumas falas, questiona-se qual a percepção de modelo de cuidado elas consideram como referência para seu acompanhamento. 
Por outro lado, o cuidado em Rede também apresenta desafios como a falta de planejamento e comunicação entre os serviços da $A B$ e a área hospitalar, não garantindo a realização de ultrassom e do parto humanizado, dentre outras necessidades sinalizadas.

Talvez o foco no modelo biomédico, que ainda persiste nos serviços de saúde, limite a ampliação do cuidado na perspectiva da integralidade que poderia proporcionar articulação entre as ações, a reflexão e autonomia para todos na tomada de decisão.

Nesse sentido, a trajetória da transcendência de sujeito, como ator do processo de produção da saúde, necessita de vontade política, conhecimento e participação social. De todos!

\section{Considerações Finais}

A pesquisa se propôs investigar a compreensão e a operacionalização da RC em uma RS do Estado de São Paulo, a partir da percepção de usuários e de profissionais de saúde, cujo desenho e trajetória percorrida possibilitou o alcance do objetivo.

A percepção das mulheres sobre as ações executadas pela RC evidenciam consultas realizadas por médicos e enfermeiros. Demonstraram satisfação com orientações realizadas, como também incompreensões acerca de informações recebidas nos serviços de saúde. Nesse sentido, é notório a necessidade de investimentos quanto as ações realizadas pelos profissionais, no sentido de possibilitar autonomia às gestantes em relação ao seu cuidado.

Quanto à percepção das equipes referente a operacionalização da $\mathrm{RC}$, identificam-se desafios referentes ao funcionamento em Rede, organização do processo de trabalho das equipes e aspectos relacionados à adesão das gestantes, reiterando assim, vulnerabilidades para um efetivo trabalho em Rede.

Ressalta-se, ainda, que a utilização em ato, em trabalho vivo, das ferramentas propostas pela Política Nacional de Humanização e Política de Humanização do Pré Natal, Parto e Nascimento, com a atuação participativa e colaborativa dos atores que produzem a saúde possibilitam melhores arranjos para o cuidado e, consequentemente, experiências mais seguras e plenas para as mulheres.

Por fim, considera-se que o método escolhido, por meio das técnicas de entrevistas e grupo focal para a coleta de dados permitiu captar de gestantes e trabalhadores dos serviços de saúde, aspectos relevantes acerca da implementação da RC, além de indicar caminhos para potencializar a gestão desse processo.

\section{REFERÊNCIAS}

Brasil. Ministério da Saúde. (2006). Portaria n. 648, de 28 de março de 2006. Aprova a Política Nacional de Atenção Básica, estabelecendo a revisão de diretrizes e normas para a organização da Atenção Básica para o Programa Saúde da Família (PSF) e o Programa Agentes Comunitários de Saúde (PACS). Diário Oficial da União, Brasília (DF); Seção 1:71.

Brasil. Ministério da Saúde. (2008). Secretaria de Atenção à Saúde. HumanizaSUS: Documento Base para gestores e trabalhadores do SUS. 4a ed. Brasília (DF): Ministério da Saúde; 72 p.

Brasil. Ministério da Saúde. (2011a). Portaria n. 1459, de 24 de junho de 2011. Institui no âmbito do Sistema Único de Saúde- SUS - a Rede Cegonha. Diário Oficial da União, Brasília (DF); 27 jun 2011; Seção 1:109.

Brasil. Ministério da Saúde. (2011b). Portaria n. 1.654, de 19 de julho de 2011. Institui, no âmbito do Sistema Único de Saúde, o Programa Nacional de Melhoria do Acesso e da Qualidade da Atenção Básica (PMAQ-AB) e o Incentivo Financeiro do PMAQ-AB, denominado Componente de Qualidade do Piso de Atenção Básica Variável - PAB Variável. Diário Oficial da União, Brasília (DF); 20 jul 2011; Seção 1:79 
Brasil. Ministério da Saúde. Secretaria de Atenção à Saúde. (2012). Departamento de Atenção Básica. Atenção ao pré-natal de baixo risco. Brasília (DF): Ministério da Saúde; 2012. 318 p.

Brasil. Ministério da Saúde. (2017). Portaria de Consolidação n. 2, de 28 de setembro de 2017. Consolidação sobre as políticas nacionais de saúde do Sistema Único de Saúde. Diário Oficial da União, 3 dez 2017; Seção Supl:61.

Brasil. Ministério da Saúde. Departamento de Atenção Básica. (2018a). Pesquisa de Histórico de Cobertura de Estratégia de Saúde da Família e Atenção Básica [Internet]. Brasília (DF): Ministério da Saúde; [citado 1 jul 2020]. Disponível em: https://www.saude.gov.br/component/tags/tag/dab

Brasil. Ministério da Saúde (2018). Secretaria de Vigilância em Saúde. DATASUS. Mortalidade no Brasil. Mortalidade neonatal p/resid [Internet]. Brasília (DF). http:tabnet.datasus.gov.br

Brasil. Ministério da Saúde. (2018b). DATASUS [Internet].[citado 6 nov 2018]. Disponível em: http://datasus.saude.gov.br

Brasil. Ministério da Saúde. (2020). Portal da Secretaria da Atenção Primária à Saúde [Internet] Brasília (DF): Ministério da Saúde; [2020] [citado 5 ago 2020]. Disponível em: https://aps.saude.gov.br

Cardelli AAM, Marrero TL, Ferrari RAP, Martins JT \& Serafim D. (2016). Expectativas e satisfação de gestantes: desvelando o cuidado pré-natal na atenção primária. Invest Educ Enferm. 2016;34(2):252-60. DOI: 10.17533/udea.iee.v34n2a04

Conselho Federal de Enfermagem. (2016). Resolução Cofen n. 516, de 23 de junho de 2016. Normatiza a atuação e a responsabilidade do enfermeiro, enfermeiro obstetra e obstetriz na assistência às gestantes, parturientes, puérperas e recém-nascidos nos serviços de obstetrícia, centros de parto normal e/ou casas de parto e outros locais onde ocorra essa assistência; estabelece critérios para registro de títulos de enfermeiro obstetra e obstetriz no âmbito do sistema Cofen/Conselhos Regionais de Enfermagem, e dá outras providências. Diário Oficial da União, Brasília (DF); 27 jun 2016; Seção 1:92

Conselho Federal de Enfermagem. (2017). Resolução Cofen n. 564, de 06 de novembro de 2017. Aprova o novo Código de Ética dos Profissionais de Enfermagem. Diário Oficial da União, Brasília (DF); 06 dez 2017. Diário Oficial da União, Brasília (DF); 27 jun 2016; Seção 1:157.

Conselho Nacional de Secretarias Municipais da Saúde. Conselho Nacional de Secretarias Municipais da Saúde [Internet]. Brasília (DF): CONASEMS; [2020] [citado 5 ago 2020]. Disponível em: https://conasems.org.br

Duarte SJH, Andrade SMO. O significado do Pré-Natal para mulheres grávidas: uma experiência no município de Campo Grande, Brasil. Saúde Soc. 2008;17(2):132-9. DOI: 10.1590/S010412902008000200013.

Elias PE, Ferreira CW, Alves MCG, Cohn A, Kishima V, Escrivão Junior A, Gomes A, Bousquat A Atenção Básica em Saúde: comparação entre PSF e UBS por estrato de exclusão social no município de São Paulo. Ciênc Saúde Colet. 2006;11(3):633-41. DOI: 10.1590/S141381232006000300012 .

Freire P. Pedagogia da autonomia: saberes necessários à prática educativa. 48a ed. Paz e Terra; 2014.

Gomes CBA, Dias RS, Silva WGB, Pacheco MAB, Sousa FGM, Loyola CMD. Consulta de enfermagem no pré-natal: narrativas de gestantes e enfermeiras. Texto Contexto Enferm. 2019;28:e20170544. DOI: 10.1590/1980-265X-TCE-2017-0544

Livramento DVP, Backes MTS, Damiani PR, Castillo LDR, Backes DS \& Simão MAS. (2019a) Percepções de gestantes acerca do cuidado pré-natal na atenção primária à saúde. Rev Gaúcha Enferm. 2019;40:e20180211. DOI: 10.1590/19831447.2019.20180211.

Livramento DVP, Backes MTS, Damiani PR, Castillo LDR, Backes DS \& Simão MAS. (2019b) Percepções de gestantes acerca do cuidado pré-natal na atenção primária à saúde. Rev Gaúcha Enferm. 2019;40:e20180211. DOI: 10.1590/19831447.2019.20180211.

Minayo MCS. (2014). O desafio do conhecimento: pesquisa qualitativa em saúde. 14a ed. Hucitec; 2014. $407 \mathrm{p}$.

Minayo MCS, organizadora. (2015). Pesquisa Social: teoria, método e criatividade. 34a ed. Editora Vozes; 2015. $108 \mathrm{p}$ 
Oliveira IG, Castro LLS, Massena AM, Santos LVF, Sousa LB, Anjos SJSB. Qualidade da consulta de enfermagem na assistência ao pré-natal de risco habitual. Rev Eletr Enf. 2017;19. DOI: 10.5216/ree.v19.40374.

Organização Mundial da Saúde. Marco para ação em educação interprofissional e prática colaborativa. Genebra (SWI): OMS; 2010. 64 p.

Orvik A, Larun L, Berland A, Ringsberg KC. Situational factors in focus group studies: A systematic review. Int J Qual Met. 2013;12(1):338-58. DOI: 10.1177/160940691301200116.

Pierantoni CR, Vianna CMM, França T, Magnago C \& Rodrigues MPS. Rotatividade da força de trabalho médica no Brasil. Saúde Debate. 2015;39(106):637-47. DOI: 10.1590/0103110420151060003006

Pires DEP, Machado RR, Soratto J, Scherer MA, Gonçalves ASR \& Trindade LL. Cargas de trabalho da enfermagem na saúde da família: implicações no acesso universal. Rev LatinoAm Enferm. 2016;24:e2677. DOI: 10.1590/1518-8345.0992.2682.

São Paulo (Estado). (2011) Comissão Inter Gestores Bipartite de São Paulo. Deliberação CIB-SP n. 36 de 21 de setembro de 2011. Constitui as Redes Regionais de Atenção à Saúde no Estado de São Paulo. Diário Oficial do Estado, São Paulo (SP); 2011 set 22; Seção 1:51.

São Paulo (Estado). (2012) Secretaria da Saúde. Deliberação CIB n. 85, de 30 de novembro de 2012. Aprovação do Plano de Ação da Rede Cegonha da RRAS 10 Marília. Diário Oficial do Estado, São Paulo (SP); 01 dez 2012; Seção 1:36.

Terra LSV, Campos GWS. Alienação do trabalho médico: tensões sobre o modelo biomédico e o gerencialismo na atenção primária. Trab Educ Saúde. 2019;17(2):e0019124. DOI: 10.1590/1981-7746-sol00191.

Vasconcellos MTL, Silva PLN, Pereira APE, Schilithz AOC, Souza Junior PRB \& Szwarcwald CL. Desenho da amostra Nascer no Brasil: Pesquisa Nacional sobre o Parto e Nascimento. Cad Saúde Pública. 2014;30(Supl 1):S49-S58. DOI: 10.1590/0102-311X00176013. 